\title{
Phytomedizin
}

\section{Efeuextrakt bei erkältungsbedingtem Husten}

Eine prospektive, offene Studie bestätigte die Wirksamkeit und Sicherheit dieser pflanzlichen Therapie.

Entzündliche Bronchialaffektionen rangieren unter den häufigsten Gründen, eine ärztliche Konsultation zu suchen. In Europa existiert eine verbreitete Tradition, Affektionen des Respirationstraktes symptomatisch mit Pflanzenextrakten zu behandeln.

\section{Pharmakokinetik}

Haeberlein und seine Mitarbeiter haben festgestellt, dass die sekretolytischen und bronchodilatativen Eigenschaften des Efeu- (Hedera helix) Extraktes auf dessen Gehalt an Saponinen zurückzuführen ist. Im Besonderen wird dem enthaltenen Alpha Hederin [1, 2] über eine inhibitorische Wirkung auf die Beta-2-Rezeptor-Endozytose eine indirekte beta-sympathomimetische Wirkung zugeschrieben [3].

\section{Wissenschaftliche Datenlage}

Die Wirksamkeit und Sicherheit von Efeuextrakt wurde durch eine Serie von klinischen Studien [4-9] bestätigt. Unterscheiden sich diese zwar in ihren Zielen, Methoden, Studienpopulationen und den behandelten Erkrankungen des Respirationstraktes, so ist innen aber doch eines gemeinsam: Sie haben Daten zur Bestätigung der Wirksamkeit dieser pflanzlichen Behandlung bei Kindern und Erwachsenen erbracht, wie sie bei anderen pflanzlichen Wirkstoffpräparationen weder so zahlreich, noch mit so signifikanter Aussage vorliegen.

\section{Die vorliegende Studie}

In der vorliegenden PostmarketingStudie [10] wurden 9657 Patienten, von denen 5181 Kinder waren, und welche alle an Husten infolge einer Erkältungskrankheit litten, mit einem Sirup behandelt, der einen Extrakt aus getrockneten Efeublättern als Wirkstoff enthielt. Nach sieben Therapie- tagen zeigten 95,1\% (Cl: 94,6-95,5) der Probanden eine Verbesserung oder gar ein Verschwinden ihrer Symptome (- Abb. 1). Auch die Sicherheit der Behandlung wurde, wie die mit 2,1\% als äußerst niedrig zu bezeichnende Inzidenz unerwünschter Ereignisse zeigte, durch die Studie bestätigt. Bei jenen Patienten, die eine Begleitmedikation erhielten, konnte gezeigt werden, dass eine zusätzliche Gabe von Antibiotika keinen zusätzlichen Benefit hinsichtlich der Wirksamkeit ergab, jedoch das relative Risiko für das Auftreten einer unerwünschten Nebenwirkung um $26 \%$ erhöhte.

\section{Fazit für die Praxis}

Zusammenfassend gelang es der vorliegenden Studie, den Stellenwert des Extrakts aus getrockneten Efeublättern in der Therapie von Patienten mit Husten bei Erkältungskrankheiten als wirksam und gut verträglich zu bestätigen: Sowohl Erwachsene als auch Kinder zeigten nach der siebentägigen Therapie eine Besserung oder Heilung ihrer Symptomatik.

\section{Literatur}

1. Bedir E, Kirmizipekmez H, Sticher O, Caliş I, (2000) Triterpene saponins from the fruits of Hedera helix. Phytochemistry 53(8):905-909

2. Trute A, Nahrstedt A (1997) In vitro antispasmodic compounds of the dry extract obtained from Hedera helix. Planta Med. 63(2):125-129

3. Hegener O, Prenner L, Runkel F, Baader SL, Kappler J, Häberlein H (2004) Dynamics of beta2-adrenergic receptor-ligand complexes on living cells. Biochemistry. 43(20): 6190-6199

4. Gulyas A, Lämmlein M (1992) Zur Behandlung von Kindern mit obstruktiver Bronchitis. Sozial Padiatr. 8:632-634

5. Laessig et al (1996) Wirksamkeit und Verträglichkeit efeuhaltiger Hustenmittel. TW Paediatr 9:489-491

6. Gulyas A, Repges R, Dethlefsen U (1997) Konsequente Therapie chronisch-obstruktiver Atemwegserkrankungen bei Kindern Atemwegs. Lungenerkrank. 2:291-294
7. Mansfeld H, Hoehre H, Repges R, Dethlefsen U (1998) Therapie des Asthma bronchiale mit Efeublätter-Trockenextrakt. MMW 140:26-30

8. Hecker M, (1999) Wirksamkeit und Verträglichkeit von Efeuextrakt bei Patienten mit Atemwegserkrankungen. NaturaMed 14:28-33

9. Hecker M, Runkel F, Voelp A (2002) Behandlung chonischer Bronchitis mit einem Spezialextrakt aus Efeublättern - multizentrische Anwendungsbeobachtung mit 1350 Patienten

10. Fazio et al (2009) Tolerance, safety and efficacy of Hedera helix extract in inflammatory bronchial diseases under clinical practice conditions: A prospective, open, multicentre postmarketing study in 9657 patients.

Phytomed. 16(1):17-24

\section{Fachkurzinformation siehe Seite A10}

Freigabecode: PROSPAN_2019_001

Paediatr. Paedolog. Austria 2019 · 54:37 https://doi.org/10.1007/s00608-0190643-4

(c) Springer-Verlag GmbH Austria, ein Teil von Springer Nature 2019

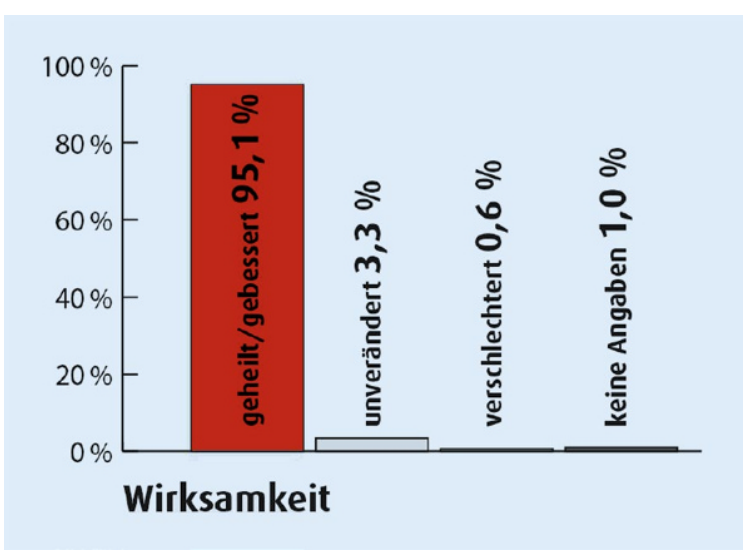

Abb. 1 A Wirksamkeit von Prospan bei 9657 Erwachsenen und Kindern [10] 